\title{
About One of a Fixed Point Theorem
}

\section{Husham Rahman Mohammed ${ }^{1}$ and Hala Abbas Mehdi ${ }^{2}$}

${ }^{1}$ Department of Mathematics, College of Computer Science and Information Technology,

University of Al-Qadisiyah, Iraq; e-mail: hishamrehman@rambler.ru

${ }^{2}$ Department of Mathematics, College of Computer Science and Information Technology,

University of Al-Qadisiyah, Iraq; e-mail: halahaider2015@gmail.com

* Corresponding author

\begin{abstract}
In this paper, we give an overview of the main directions in the theory of fixed points of multivalued mappings. We prove a fixed point theorem of multivalued mapping and the following lemma has important role in the proof of main theorem.
\end{abstract}

\section{Introduction and Preliminaries}

The study of the immobile problem is important for applications such as the points of multivalued mappings are devoted, starting with the works of von Neumann [3], Kakutani [2], Wallace [4], Eilenberg-Montgomery [1] and other.

Let $X, Y$ be metric spaces, $K$ be a compact subset in $X$ and $f: X \rightarrow Y$ be a continuous mapping.

Lemma 1. For any $\delta>0$, there exists $\varepsilon>0$ such that as soon as $\rho\left(x^{\prime}, x^{\prime \prime}\right)<\varepsilon$ and $x^{\prime}, x^{\prime \prime} \in U_{\varepsilon}(K)$, then $\rho\left(f\left(x^{\prime}\right), f\left(x^{\prime \prime}\right)\right)<\delta$.

Received: August 26, 2019; Revised: October 13, 2019; Accepted: October 18, 2019

2010 Mathematics Subject Classification: 46E15.

Keywords and phrases: multivalued map, $\delta$-approximation, absolutely continuous, super positionally approximable multivalued map (SA-map), Michael's correct system AM $(Y)$, fixed point.

Copyright (C) 2019 Husham Rahman Mohammed and Hala Abbas Mehdi. This is an open access article distributed under the Creative Commons Attribution License, which permits unrestricted use, distribution, and reproduction in any medium, provided the original work is properly cited. 
Proof. Let $\delta$ be an arbitrary positive number and the set $K$ be compact. Then, there exists $\eta>0$ such that for any $y_{1}, y_{2} \in K$ as soon as $\rho\left(y_{1}, y_{2}\right)<\eta$, we have

$$
\rho\left(f\left(y_{1}\right), f\left(y_{2}\right)\right)<\frac{\delta}{3} .
$$

Let $x$ be an arbitrary point of $K$. Then, by the continuity of the mapping $f$, there is an open neighborhood $U_{\lambda(x)}(x)$ of point $x$ such that for any $x_{1}, x_{2} \in U_{\lambda(x)}(x)$, the inequality

$$
\rho\left(f\left(x_{1}\right), f\left(x_{2}\right)\right)<\frac{\delta}{3} .
$$

Without loss of generality, we can assume that $0<\lambda(x)<\frac{\eta}{3}$.

It is obvious that the family $\left\{U_{\lambda(x)}(x)\right\}_{x \in X}$ forms an open cover of the space $X$.

Let $U=\bigcup_{x \in K} U_{\lambda(x)}(x)$, this set is open and contains $K$. The set $K$ is a compact, and there is a number $\eta_{1}>0$ such that $U \eta_{1}(K) \subset U$. Consider $\varepsilon=\min \left\{\frac{\eta}{3}, \eta_{1}\right\}$. We show that this number satisfies the conditions of the lemma.

Let $x^{\prime}, x^{\prime \prime} \in U_{\varepsilon}(K)$. Then there are $y_{1}, y_{2} \in K$ such that $x^{\prime} \in U_{\lambda\left(y_{1}\right)}\left(y_{1}\right)$, $x^{\prime \prime} \in U_{\lambda\left(y_{2}\right)}\left(y_{2}\right)$. If $\rho\left(x^{\prime}, x^{\prime \prime}\right)<\varepsilon$, then

$$
\rho\left(f\left(x^{\prime}\right), f\left(x^{\prime \prime}\right)\right) \leq \rho\left(f\left(x^{\prime}\right), f\left(y_{1}\right)\right)+\rho\left(f\left(y_{1}\right), f\left(y_{2}\right)\right)+\rho\left(f\left(y_{2}\right), f\left(x^{\prime \prime}\right)\right) .
$$

We note that

$$
\rho\left(f\left(x^{\prime}\right), f\left(y_{1}\right)\right)<\frac{\delta}{3}
$$

and

$$
\rho\left(f\left(y_{2}\right), f\left(x^{\prime \prime}\right)\right)<\frac{\delta}{3} \text {. }
$$

The values of $\rho\left(f\left(y_{1}\right), f\left(y_{2}\right)\right)$, we note that

$$
\rho\left(y_{1}, y_{2}\right) \leq \rho\left(y_{1}, x^{\prime}\right)+\rho\left(x^{\prime}, x^{\prime \prime}\right)+\rho\left(x^{\prime \prime}, y_{2}\right)<\lambda\left(y_{1}\right)+\varepsilon+\lambda\left(y_{2}\right)<\eta,
$$


and

$$
\rho\left(f\left(y_{1}\right), f\left(y_{2}\right)\right)<\frac{\delta}{3} .
$$

Therefore,

$$
\rho\left(f\left(x^{\prime}\right), f\left(x^{\prime \prime}\right)\right)<\delta
$$

The lemma is proved.

Lemma 2. Let $T$ be a closed convex bounded subset of the Banach space E, $F: T \rightarrow K(E)$ be completely continuous multivalued mapping.

If for any $\delta>0$ there is an unambiguous $\delta$-approximation of $f_{\delta}$ map $F$ having a fixed point, then the map $F$ also has a fixed point.

Proof. Consider an arbitrary sequence of positive integers $\delta_{n}$ that tends to zero. Let $f_{\delta_{1}}=f_{n}$ be a unique $\delta_{n}$-approximation satisfying the conditions of the lemma. Let $x_{n}$ - the fixed point of the map is $f_{n}$, i.e., $f_{n}\left(x_{n}\right)=x_{n}$. By definition of a unique $\delta$-approximation, there are points $x_{n}^{\prime}$ and $y_{n}$ such that

$$
\left\|x_{n}-x_{n}^{\prime}\right\|<\delta_{n}, y_{n} \in F\left(x_{n}^{\prime}\right) \text { and }\left\|x_{n}-y_{n}\right\|<\delta_{n} .
$$

Since the map $F$ is completely continuous, the set $F(T)$ is relatively compact. Using the fact that the points $y_{n} \in F(T)$, and without limitation of generality, we assume that the sequence $\left\{y_{n}\right\}$ converges to the point $y$. Hence the sequences $\left\{x_{n}\right\}$ and $\left\{x_{n}^{\prime}\right\}$ also converge to this point. The sequence $\left\{x_{n}\right\} \subset T$, the point $y$ also belongs to this set. Now the statement of the lemma follows from the closure graphics of an upper semicontinuous of a multivalued map $F$.

Let $X$ be a metric space and $\Phi: X \rightarrow K(E)$ be an upper semicontinuous multivalued map.

Definition 1. A multivalued map $\Phi$ is called a super positionally approximable multivalued map (SA-map), if there is metric space $Y$, the Michael's correct system $A M(Y)$ in space $Y$, semi-continuous of a upper semi-continuous of a multivalued map $F: X \rightarrow A M(Y)$, a continuous uniquely map $p: Y \rightarrow E$ such that for any point $x \in X$ there is true equality $\Phi(x)=p(F(X))$. 
A multivalued map $\Phi$ is called a completely continuous $S A$ map if the multivalued map $F: X \rightarrow A M(E)$ is completely continuous.

\section{Main Result}

From the above, we drive the main theorem which can be regarded as an extension of the most fixed point theorem.

Theorem. Let $T$ be a closed convex bounded subset of the Banach space $E$ and $\Phi: T \rightarrow K(E)$ be a multivalued completely continuous SA-map. If $\Phi(T) \subset T$, then the map $\Phi$ has a fixed point.

Proof. Let $\tau: E \rightarrow T$ be a continuous retraction of space $E$ by $T$, and $\eta$ be an arbitrary positive number. By the boundedness of the set $T$, there is a number $R>0$, such that $U_{\eta}(T) \subset B_{R}[0]$.

Let $\Phi=p_{0} F$, where $p$ and $F$ are from Definition 1. Consider the continuous map $p_{1}=\tau$ and $p: Y \rightarrow T$, where $K=\overline{F(T)} \subset Y$. By the impact of the complete continuity of the map $F$, the set $K$ is compact, there for the impact of Lemma 1 for any $\delta \in(0, \eta)$ there is $\varepsilon>0$ such that as soon as $\rho\left(x^{\prime}, x^{\prime \prime}\right)<\varepsilon$ and $x^{\prime}, x^{\prime \prime} \in U_{\varepsilon}(K)$, then $\rho\left(p_{1}\left(x^{\prime}\right), p_{1}\left(x^{\prime \prime}\right)\right)<\delta$. Without limitation of generality, we assume that $\varepsilon<\delta$, which results that in a multivalued mapping $F$ there is a completely continuous $\varepsilon$-approximation of $f: T \rightarrow Y$. We show that the composition $f_{1}=p_{1} . f$ is a completely continuous $\delta$-approximation of the map $\Phi_{1}=p_{1} F$. We assume that, let $x$ be an arbitrary point of $T$, and according to the definition of $\varepsilon$-approximation, there exists a point $x^{\prime} \in T, y \in F\left(x^{\prime}\right)$ such that $\left\|x-x^{\prime}\right\|<\varepsilon$ and $\rho(f(x) ; y)<\varepsilon$, therefore, $f(x) \in U_{\varepsilon}(K)$. Then

$$
\left\|p_{1}(f(x))-p_{1}(y)\right\|<\delta .
$$

Since $p_{1}(y) \in p_{1}\left(F\left(x^{\prime}\right)\right)=\Phi_{1}\left(x^{\prime}\right), \quad f_{1}$ which results a continuous $\delta$-approximation.

From the compactness of the $f_{1}$-map and the compactness of the $f$-map, we note that $f_{1}\left(B_{R}[0]\right) \subset B_{R}[0]$. And, for $x \in B_{R}[0]$ we have

$$
f_{1}(x)=\tau(p(f(x))) \in T \subset B_{R}[0] .
$$


Thus according to Schauder's theorem, the map $f_{1}$ has a fixed point, and by Lemma 2 , the multivalued map $\Phi_{1}$ has a fixed point. We let $x_{*} \in \Phi_{1}\left(x_{*}\right)=\tau\left(\Phi\left(x_{*}\right)\right)$. Since $x_{*} \in T$, we have $\tau\left(\Phi\left(x_{*}\right)\right)=\Phi\left(x_{*}\right)$. This proves the theorem.

\section{References}

[1] S. Eilenberg and D. Montgomery, Fixed point theorems for multi-valued transformations, Amer. J. Math. 68 (1946), 214-222. https://doi.org/10.2307/2371832

[2] S. Kakutani, A generalization of Brouwer's fixed point theorem, Duke Math. J. 8 (1941), 457-459. https://doi.org/10.1215/S0012-7094-41-00838-4

[3] J. von Neumann, Über ein Ökonomisches Gleichungssystem und eine Verallgemeinung des Brouwerschen Fixpunktsatzes, Ergebnisse eines Mathematischen Kolloquiums 8 (1937), 73-83.

[4] A. D. Wallace, A fixed-point theorem for trees, Bull. Amer. Math. Soc. 47 (1941), 757760. https://doi.org/10.1090/S0002-9904-1941-07556-7 